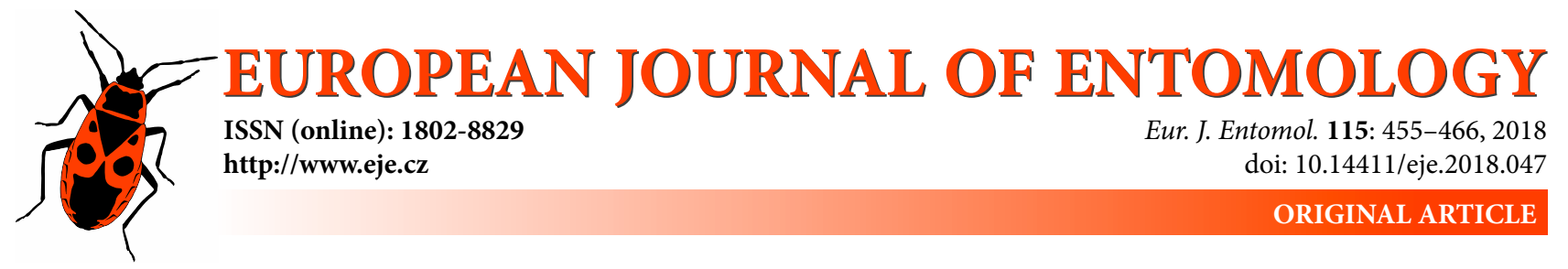

\title{
Mandible morphology reflects the type of male antagonism in the ant genus Cardiocondyla (Hymenoptera: Formicidae)
}

\author{
Christine V. SCHMidT and JüRgen HEINZE \\ Zoology / Evolutionary Biology, University of Regensburg, Universitätsstraße 31, 93053 Regensburg, Germany; \\ e-mails: christine.schmidt@biologie.uni-regensburg.de, juergen.heinze@biologie.uni-regensburg.de
}

Key words. Hymenoptera, Formicidae, Cardiocondyla, ergatoid males, morphology, mandibles, male antagonism

\begin{abstract}
The ant genus Cardiocondyla is characterized by wingless, ergatoid males, which in some species replace the typical winged male. Depending on species, ergatoid males engage in lethal fighting for access to sexual females, establish territories within their nests, or are mutually tolerant. Here we investigate, whether the morphology of ergatoid males and in particular the shape of their mandibles reflect phylogeny or male behaviour. In contrast to the worker mandible, which is relatively similar in shape in the ten species examined, mandibles of ergatoid males show a great variation in size and shape not only between the two clades of Cardiocondyla but also among species belonging to Cardiocondyla clade A. This diversity appears to reflect the diverse reproductive tactics of ergatoid males of different species, with extremely long, anteriorly oriented mandibles associated with indirect killing of adult competitors, while particularly short mandibles possibly constitute an adaptation to a tactic in which ergatoid males crush the cuticle of young rivals.
\end{abstract}

\section{INTRODUCTION}

The genus Cardiocondyla Emery is unusual among ants in having two different male phenotypes, winged males and ergatoid males. Winged males are typical of most ant species but occur in less than $25 \%$ of the species of Cardiocondyla examined (Heinze, 2017). With fully developed wings, large eyes, ocelli and a bulky thorax they are well adapted to dispersal, searching for female sexuals and mating away from their natal nests. Ergatoid males occur in all the species of Cardiocondyla examined. They stay inside the maternal nest and locally mate with available female sexuals (Heinze, 2017). Their entirely non-dispersing life cycle is reflected in a superficially "worker-like" morphology: ergatoid males are wingless, have small eyes and lack ocelli. They may be relatively long-lived (up to one year; Yamauchi et al., 2006), and, in contrast to all other males of social Hymenoptera, are characterized by life-long spermatogenesis (Heinze \& Hölldobler, 1993).

Winged males of Cardiocondyla are docile and do not engage actively in fighting for access to female sexuals. In contrast, competition among ergatoid males ranges from complete mutual tolerance (Schrempf et al., 2005; Schrempf \& Heinze, 2007), defense of small territories in the nest (Frohschammer \& Heinze, 2009; Jacobs \& Heinze, 2017) to regular killing of immature rivals and fatal fighting among mature males (e.g., Kinomura \& Yamauchi, 1987; Yamauchi \& Kawase, 1992). Mandibles are the principal tool used in male fighting and different fighting tech- niques are clearly associated with differences in mandible morphology. The phylogeny of Cardiocondyla indicates a dichotomy between two clades (Oettler et al., 2010), which according to Ward et al. (2014) diverged approximately 20 million years ago. In one branch (clade A), ergatoid males use their "sickle-shaped" mandibles to puncture the soft cuticle of young males ("callows"). In addition, they grasp older, sclerotized rivals and daub them with hindgut secretion, which elicits aggression by workers towards the besmeared individual (Kinomura \& Yamauchi, 1987; Stuart et al., 1987; Yamauchi \& Kawase, 1992). In the other branch, clade B, ergatoid males have "shear-shaped" mandibles superficially similar to those of workers. They eliminate unsclerotized callows by crushing or dismembering them (Heinze et al., 1993, 1998; Frohschammer \& Heinze, 2009) but are rarely capable of injuring competitors with their mandibles once the cuticle of the latter has hardened. Nests of species in clade B may therefore contain many adult males, which typically do not show agonistic behaviour (Heinze et al., 1993, 1998). In one particular lineage of clade B male fighting apparently has been completely lost (e.g., Schrempf et al., 2005; Lenoir et al., 2007), with male territoriality probably being an intermediate stage between fighting and tolerance (Frohschammer \& Heinze, 2009; Jacobs \& Heinze, 2017).

Here we compare males and workers of several species of Cardiocondyla and investigate whether it is possible to extrapolate male morphology from that of workers, which 
is known for many more species (Seifert, 2003). More importantly, mandibles of most Cardiocondyla males have only been described superficially (e.g., Kugler, 1983; Terayama, 1999). We therefore describe the morphology of the mandibles of ergatoid Cardiocondyla males with different reproductive life histories. By doing so, we aim to reveal possible correlations between male morphology and reproductive tactics.

\section{MATERIAL AND METHODS}

\section{Examined species}

For the morphometric study, specimens of eight different species (C. "argyrotricha" - a taxon currently being formally described by B. Seifert, C. batesii Forel, C. elegans Emery, C. kagutsuchi Terayama, C. minutior Forel, C. nigrocerea Karavaiev, C. obscurior Wheeler, C. venustula Wheeler; Table 1) belonging to different species groups (Seifert, 2003) were examined. Morphometric measurements of both ergatoid males and workers were recorded, except for $C$. elegans, where only ergatoid males were available (see Table 1). In addition, mandible morphology was qualitatively studied using a Wild M10 stereomicroscope and/or by scanning electron microscopy in the above mentioned species and two additional species [C. tjibodana Karavaiev, $C$. wroughtonii (Forel)]. Mandibles of winged males were qualitatively studied in four species (C. minutior, C. obscurior, C. tjibodana, C. wroughtonii).

\section{Sample preparation and assessment of morphological characters}

Ants were collected either in the field or from laboratory colonies and kept in $70 \%$ ethanol in small plastic bottles. Whole specimens were dehydrated in an ascending series of alcohol $(80 \%$, $90 \%, 99.6 \%$ ethanol). Seven morphological characters were measured on dehydrated specimens mounted on conductive doublesided adhesive carbon tabs using a Wild M10 stereomicroscope (Leica Microsystems) and an ocular micrometer. Definition of numeric characters (mostly according to Seifert, 2003): CL maximum dorsal cephalic length in median line, excavations on occiput and/or clypeus reduce CL; CW - cephalic width; across and including the eyes; ML - length of mesosoma; measured in lateral view from the caudalmost portion of propodeum to the anteriormost point of the anterior pronotal slope; $\mathrm{MW}$ - maximum width of mesosoma; PEW - maximum width of petiole; PPW - maximum width of post-petiole. PEW and PPW could not be measured in ergatoid males of $C$. venustula because of their dense pubescence, which obscured the borders of the body. Cephalic size CS was used as an indicator of body size (see Seifert, 2003) and calculated as $(\mathrm{CL}+\mathrm{CW}) / 2$. After measurement of the above mentioned characters, mandibles were removed and mounted on double-sided adhesive tape in a ventral view to measure mandible length MDL: distance between the ventral condylus and the tip of the apical tooth (shear-shaped mandibles of ergatoid males and mandibles of workers) or the tip of the mandible (sickle-shaped mandibles of ergatoid males). We assume that the landmarks chosen are homologous in the two types of mandibles. A mean measurement error of about $1.4 \mu \mathrm{m}$ was calculated based on three measurements for small structures (e.g., PEW, PPW) and of about $2.5 \mu \mathrm{m}$ for larger structures (e.g., ML). To allow for comparison within and between species, characters were divided by CS. In order to describe the morphology of mandibles, complete heads and detached mandibles of additional worker and male specimens were removed and mounted in different views (dorsal, ventral, mesal and lateral) on metal stubs with double-sided adhesive carbon pads and air-dried for at least $48 \mathrm{~h}$. After this procedure, samples were coated with a mixture of gold-palladium in a sputter-coater (Fisons Instruments, UK) and examined using a DSM 950 Zeiss Scanning Electron Microscope. The terminology of the morphology of mandibles largely follows Ettershank (1966) and Gotwald (1969), except for mandibles of ergatoid and winged males belonging to Cardiocondyla clade A (C. obscurior, C. wroughtonii, $C$. nigrocerea and $C$. "argyrotricha"). Here, we use "dorsal margin" because of the derived morphology of these mandibles, which makes it difficult to homologize this margin with the "basal margin" of typical triangular mandibles. Furthermore, we avoid the term "external margin" in the description of Cardiocondyla mandibles, as it is usually used in in-situ descriptions, and corresponds to the lateral margins seen in full-face view of ants. In our description of detached mandibles, we use the term "ventral margin" to describe the margin running ventrally from the ventralmost point of the articulation with the head capsule to the anteriormost point of the mandible.

We used the software IBM SPSS Statistics 23 (IBM Corporation) and R 3.2.1 (R Development Core Team, 2015) to perform statistical analyses, including a Principal Component Analysis (PCA), to examine the variation in the morphometric data of six characters within and among species: CW, CL, MW, PEW, PPW, ML. We did not include mandible length (MDL) in the PCA. This character clearly distinguishes ergatoid males of clades A and B (Oettler et al., 2010) and might therefore have obscured other morphological trends. To take body size into account in the PCA the six metric characters were divided by CS. PCA results were

Table 1. Species of the two clades examined, presence of male phenotypes and reproductive tactics.

\begin{tabular}{|c|c|c|c|c|}
\hline Species group & Species & Collection site & Male phenotype & Reproductive tactic \\
\hline \multicolumn{5}{|c|}{ Clade A: sickle-shaped mandibles } \\
\hline C. wheeleri group & C. nigrocerea* & Pulau Obi, Maluku Utara, Indonesia & ergatoid & besmearing callows but rarely adult males \\
\hline C. "argentea" group ${ }^{1}$ & C. "argyrotricha"* & Los Baños, Philippines & ergatoid & besmearing callows ${ }^{2}$ \\
\hline C. wroughtonii group & $\begin{array}{l}\text { C. wroughtonii } \\
\text { C. obscurior }\end{array}$ & $\begin{array}{l}\text { Limay, Bataan, Philippines } \\
\text { Ilhéus, Bahia, Brazil }\end{array}$ & $\begin{array}{l}\text { ergatoid, winged } \\
\text { ergatoid, winged }\end{array}$ & $\begin{array}{l}\text { piercing callows and besmearing adults } \\
\text { piercing callows and besmearing adults }\end{array}$ \\
\hline \multicolumn{5}{|c|}{ Clade B: shear-shaped mandibles } \\
\hline \multirow[t]{2}{*}{ C. minutior group } & C. minutior ${ }^{*}$ & Okinawa, Japan & ergatoid, winged & crushing callow cuticle \\
\hline & C. tjibodana & Gua Londron, Indonesia & ergatoid, winged & crushing callow cuticle \\
\hline C. nuda group & C. kagutsuchi* & Okinawa, Japan & ergatoid & crushing callow cuticle \\
\hline C. shuckardi group & C. venustula** & Kaua'i, Hawaii, USA & ergatoid & $\begin{array}{l}\text { crushing callow cuticle, } \\
\text { adult fighting and besmearing }\end{array}$ \\
\hline C. batesii group & C. batesii ${ }^{*}$ & Padul, Spain & ergatoid & tolerant \\
\hline C. elegans group & C. elegans ${ }^{*}$ & Chemilly, Allier, France & ergatoid & tolerant \\
\hline
\end{tabular}

* measurement of seven morphological characters; ${ }^{* *}$ measurement of five morphological characters; ${ }^{1} \mathrm{C}$. "argentea" and C. "argyrotricha" are the preliminary names for taxa currently being described by B. Seifert; ${ }^{2}$ authors' assumption based on occasional observations of lab-reared colonies. 
plotted in two-dimensional PCA scatter plots. We used the first two principal components (PCs), which accounted for over 91\% of the total variation.

\section{RESULTS}

\section{Morphometric data}

Principal component analysis of data on six morphological characters of six to 12 workers and ergatoid males of each of six species (C. obscurior, C. nigrocerea, C. "argyrotricha", C. minutior, C. kagutsuchi, C. batesii) produced two axes, which together explained $91.42 \%$ of the total variance (Fig. 1). The first axis accounted for $75.83 \%$ with nearly equal contributions of all the characters measured (CW: 19.42\%, CL: 13.54\%, MW: 18.24\%, PEW: 14.14\%, PPW: $16.26 \%$, ML: $18.40 \%$ ). The second axis explained $15.59 \%$ and indicated a relationship with CL, PEW and PPW (CL: 36.79\%, PEW: 33.48\%, PPW: 14.01\%). Ergatoid males were mainly separated from conspecific workers by PC 2 .

The diverse directions of the vectors between the centroids of worker and male measurements indicate that as it is based on so few species it is not possible to make more precise predictions about male morphology.

All characters differ significantly between males and workers in the species examined (two-sample t-test/ Welch's t-test, $\mathrm{p}<0.05)$, except for MW/CS of $C$. minutior $(t=-1.792, p=0.104)$. Comparison of the data on seven characters (including mandible length) revealed several differences between workers and males in seven species of Cardiocondyla. CL/CW is generally smaller in ergatoid males (Fig. 2A), while PEW/CS (Fig. 2B), PPW/CS (Fig. 2C) and MW/CS (Fig. 2D) are larger in ergatoid males.

Concerning the morphology of mandibles, MDL/CS is generally larger in ergatoid males with sickle-shaped mandibles (clade $\mathrm{A} ;>0.5)$ than in workers $(<0.5)$. In contrast,

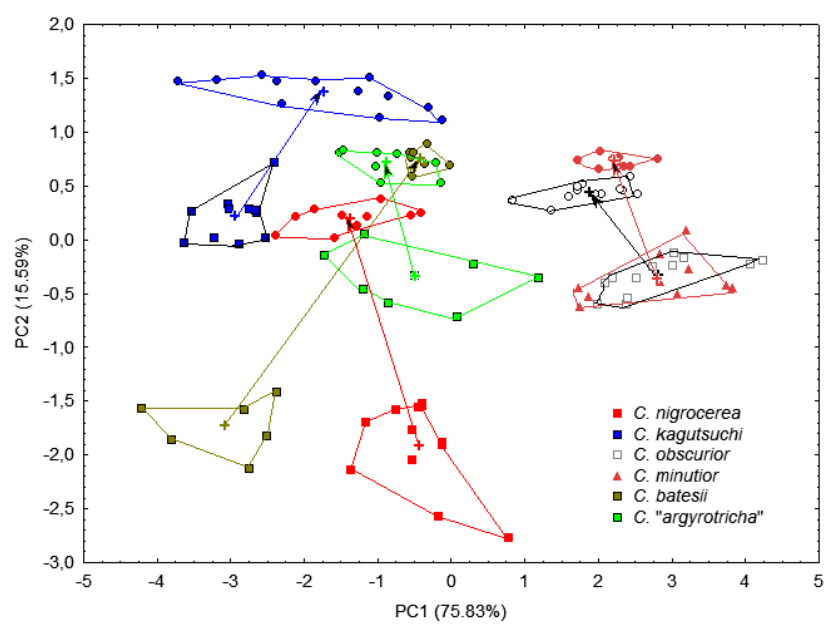

Fig. 1. Analysis of Principal Components of the morphometric data of ergatoid males (squares, triangle) and workers (circles) of six species of Cardiocondyla.

in clade $\mathrm{B} \mathrm{MDL} / \mathrm{CS}$ is smaller for ergatoid males with shear-shaped mandibles $(<0.5)$ than in workers $(>0.5)$ (Fig. 2E), except for C. venustula (MDL/CS $>0.5$ in both workers and ergatoid males, see Table 4). Sickle-shaped mandibles of ergatoid males in clade A are longer than those of conspecific workers, while shear-shaped mandibles of ergatoid males in clade B are shorter, except in $C$. venustula (Tables 2-4). The length of the mandible is significantly positively correlated with body size (CS) both in workers and ergatoid males, except in the ergatoid males of C. kagutsuchi (Table 2-4).

\section{Morphology of mandibles of ten species of Cardiocondyla}

Trulleum and mandalus were present in workers, ergatoid males and winged males of all the species examined.

Table 2. Measurement data of workers and males of species of the Cardiocondyla wroughtonii, C. "argyrotricha", and C. nigrocerea species-groups; arithmetic mean \pm standard deviation [range]. CL - cephalic length; CW - cephalic width; ML - mesosoma length; MW mesosoma width; PEW - width of petiole; PPW - width of postpetiole.

\begin{tabular}{|c|c|c|c|c|c|c|}
\hline Species & C. nigrocerea & C. nigrocerea & C. "argyrotricha" & C. "argyrotricha" & C. obscurior & C. obscurior \\
\hline caste & $\begin{array}{l}\text { worker } \\
{[N=10]}\end{array}$ & $\begin{array}{l}\text { ergatoid male } \\
\qquad[N=12]\end{array}$ & $\begin{array}{l}\text { worker } \\
{[\mathrm{N}=8]}\end{array}$ & $\begin{array}{l}\text { ergatoid male } \\
\qquad[N=8]\end{array}$ & $\begin{array}{l}\text { worker } \\
{[N=12]}\end{array}$ & $\begin{array}{c}\text { ergatoid male } \\
\qquad[N=12]\end{array}$ \\
\hline CS & $\begin{array}{c}500 \pm 14 \\
{[475,519]}\end{array}$ & $\begin{array}{c}417 \pm 19 \\
{[369,443]}\end{array}$ & $\begin{array}{c}515 \pm 13 \\
{[495,535]}\end{array}$ & $\begin{array}{c}472 \pm 23 \\
{[441,506]}\end{array}$ & $\begin{array}{c}441 \pm 10 \\
{[427,465]}\end{array}$ & $\begin{array}{c}379 \pm 13 \\
{[351,395]}\end{array}$ \\
\hline CL/CW & $\begin{array}{l}1.150 \pm 0.014 \\
{[1.124,1.168]}\end{array}$ & $\begin{array}{l}0.996 \pm 0.036 \\
{[0.924,1.043]}\end{array}$ & $\begin{array}{l}1.117 \pm 0.010 \\
{[1.100,1.134]}\end{array}$ & $\begin{array}{l}1.040 \pm 0.025 \\
{[1.004,1.078]}\end{array}$ & $\begin{array}{c}1.108 \pm 0.017 \\
{[1.078,1.149]}\end{array}$ & $\begin{array}{l}1.064 \pm 0.024 \\
{[1.021,1.111]}\end{array}$ \\
\hline MW/CS & $\begin{array}{l}0.637 \pm 0.014 \\
{[0.615,0.653]}\end{array}$ & $\begin{array}{l}0.816 \pm 0.026 \\
{[0.754,0.861]}\end{array}$ & $\begin{array}{l}0.677 \pm 0.010 \\
{[0.661,0.691]}\end{array}$ & $\begin{array}{l}0.744 \pm 0.029 \\
{[0.688,0.776]}\end{array}$ & $\begin{array}{l}0,599 \pm 0.009 \\
{[0.590,0.611]}\end{array}$ & $\begin{array}{l}0.708 \pm 0.025 \\
{[0.673,0.750]}\end{array}$ \\
\hline ML/CS & $\begin{array}{l}1.325 \pm 0.018 \\
{[1.292,1.343]}\end{array}$ & $\begin{array}{l}1.413 \pm 0.035 \\
{[1.356,1.500]}\end{array}$ & $\begin{array}{l}1.172 \pm 0.032 \\
{[1.119,1.203]}\end{array}$ & $\begin{array}{c}1.237 \pm 0.034 \\
{[1.165,1.270]}\end{array}$ & $\begin{array}{l}1.201 \pm 0.016 \\
{[1.177,1.225]}\end{array}$ & $\begin{array}{l}1.384 \pm 0.033 \\
{[1.318,1.436]}\end{array}$ \\
\hline PEW/CS & $\begin{array}{l}0.313 \pm 0.011 \\
{[0.299,0.331]}\end{array}$ & $\begin{array}{l}0.443 \pm 0.029 \\
{[0.405,0.505]}\end{array}$ & $\begin{array}{l}0.301 \pm 0.009 \\
{[0.291,0.318]}\end{array}$ & $\begin{array}{l}0.353 \pm 0.015 \\
{[0.333,0.372]}\end{array}$ & $\begin{array}{l}0.290 \pm 0.011 \\
{[0.275,0.312]}\end{array}$ & $\begin{array}{l}0.316 \pm 0.017 \\
{[0.291,0.340]}\end{array}$ \\
\hline PPW/CS & $\begin{array}{l}0.578 \pm 0.008 \\
{[0.565,0.592]}\end{array}$ & $\begin{array}{l}0.690 \pm 0.048 \\
{[0.641,0.801]}\end{array}$ & $\begin{array}{l}0.428 \pm 0.008 \\
{[0.416,0.436]}\end{array}$ & $\begin{array}{l}0.489 \pm 0.020 \\
{[0.465,0.526]}\end{array}$ & $\begin{array}{l}0.455 \pm 0.008 \\
{[0.442,0.469]}\end{array}$ & $\begin{array}{l}0.531 \pm 0.023 \\
{[0.494,0.567]}\end{array}$ \\
\hline MDL/CS & $\begin{array}{l}0.478 \pm 0.005 \\
{[0.472,0.490]}\end{array}$ & $\begin{array}{l}0.585 \pm 0.021 \\
{[0.560,0.632]}\end{array}$ & $\begin{array}{l}0.480 \pm 0.007 \\
{[0.468,0.492]}\end{array}$ & $\begin{array}{l}0.558 \pm 0.009 \\
{[0.546,0.570]}\end{array}$ & $\begin{array}{l}0.483 \pm 0.003 \\
{[0.478,0.490]}\end{array}$ & $\begin{array}{l}0.789 \pm 0.027 \\
{[0.728,0.825]}\end{array}$ \\
\hline MDL $[\mu \mathrm{m}]$ & $\begin{array}{c}239 \pm 7 \\
{[227,249]}\end{array}$ & $\begin{array}{c}243 \pm 5 \\
{[233,251]}\end{array}$ & $\begin{array}{c}247 \pm 8 \\
{[235,263]}\end{array}$ & $\begin{array}{c}263 \pm 11 \\
{[246,276]}\end{array}$ & $\begin{array}{c}213 \pm 5 \\
{[204,223]}\end{array}$ & $\begin{array}{c}299 \pm 19 \\
{[255,320]}\end{array}$ \\
\hline $\begin{array}{l}\text { Pearson's correlation } \\
\text { (MDL-CS) }\end{array}$ & $0.924^{\star *}$ & $0.728^{* *}$ & $0.905^{\star \star}$ & $0.944^{\star *}$ & $0.952^{* \star}$ & $0.932^{\star *}$ \\
\hline
\end{tabular}

** 0.01 significance level. 

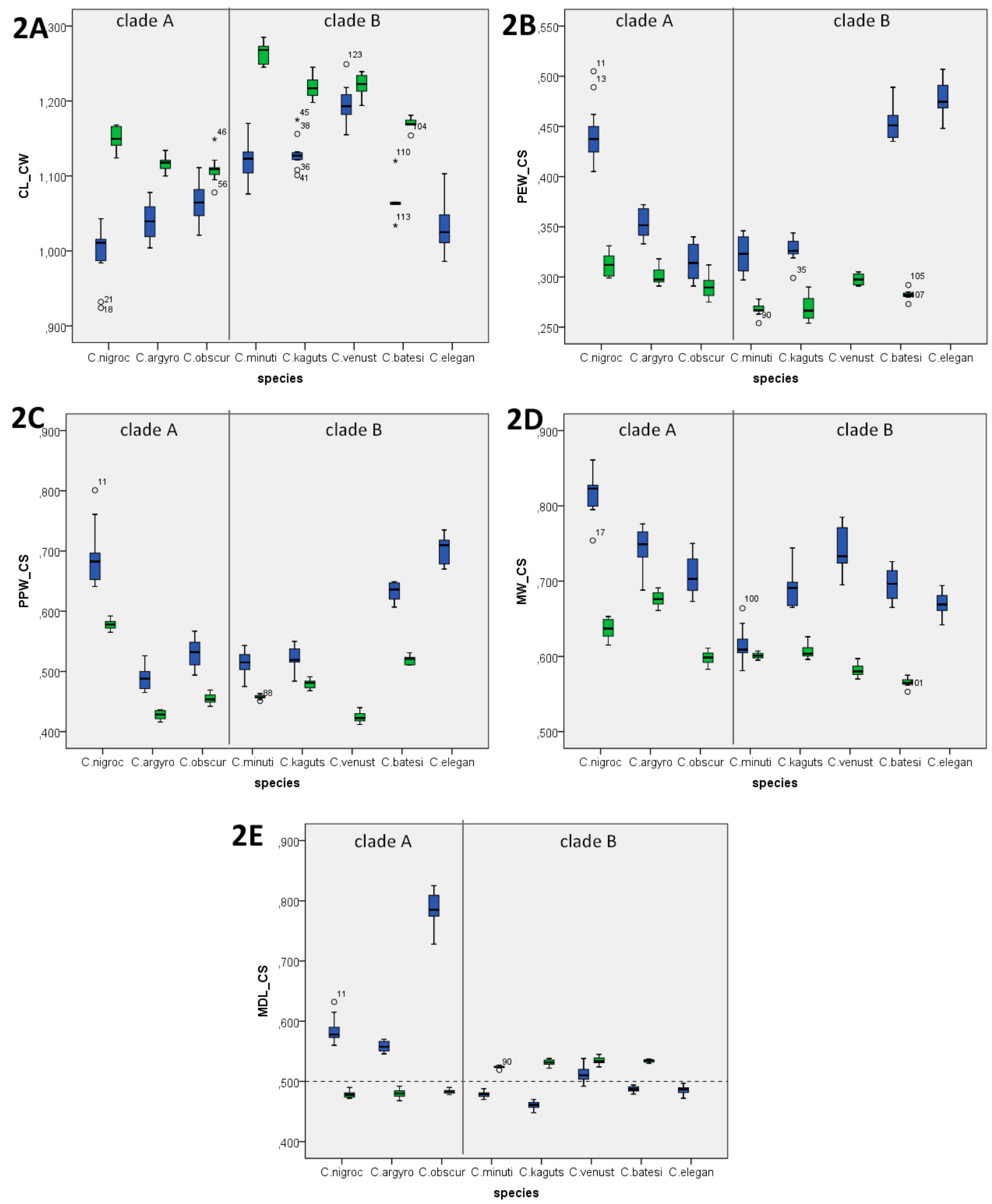

Fig. 2. Morphometric data of several characters of ergatoid males (blue plots) and workers (green plots) of eight species of Cardiocondyla. Medians, quartiles and ranges are given. A - CL/CW, B - PEW/CS, C - PPW/CS, D - MW/CS, E - MDL/CS. Characters of males and workers of all examined species are significantly different (two-sample t-test/ Welch's t-test, $p<0.05$ ), except for MW/CS of $C$. minutior, $\mathrm{t}=-1.792, \mathrm{p}=0.104)$.

Mandibles distally curved inwards and are generally opposable.

\section{Mandibles of workers}

There is little morphological variation among the species in the mandibles of workers. They have a clear basal angle and thus well-defined basal and masticatory margins (Fig. 3A). Masticatory margin bears five teeth, two apical teeth are enlarged and the apical tooth is the largest. In C. nigrocerea and C. "argyrotricha" masticatory teeth decrease in size from apical to basal (Fig. 3D), but the prebasal tooth 
Table 3. Measurement data of workers and males of species of the C. minutior and C. nuda species-groups; arithmetic mean \pm standard deviation [range]. CL - cephalic length; CW - cephalic width; ML mesosoma length; MW - mesosoma width; PEW - width of petiole; PPW - width of postpetiole.

\begin{tabular}{|c|c|c|c|c|}
\hline Species & C. minutior & C. minutior & C. kagutsuchi & C. kagutsuchi \\
\hline caste & $\begin{array}{l}\text { worker } \\
{[N=9]}\end{array}$ & $\begin{array}{l}\text { ergatoid male } \\
\qquad[N=10]\end{array}$ & $\begin{array}{l}\text { worker } \\
{[\mathrm{N}=12]}\end{array}$ & $\begin{array}{c}\text { ergatoid male } \\
\qquad[N=11]\end{array}$ \\
\hline CS & $\begin{array}{c}435 \pm 6 \\
{[422,448]}\end{array}$ & $\begin{array}{c}406 \pm 16 \\
{[379,425]}\end{array}$ & $\begin{array}{c}540 \pm 29 \\
{[495,586]}\end{array}$ & $\begin{array}{c}550 \pm 7 \\
{[539,564]}\end{array}$ \\
\hline CL/CW & $\begin{array}{l}1.263 \pm 0.014 \\
{[1.245,1.285]}\end{array}$ & $\begin{array}{c}1.119 \pm 0.026 \\
{[1.076,1.170]}\end{array}$ & $\begin{array}{l}1.219 \pm 0.014 \\
{[1.198,1.245]}\end{array}$ & $\begin{array}{l}1,130 \pm 0.021 \\
{[1.101,1.175]}\end{array}$ \\
\hline MW/CS & $\begin{array}{l}0.601 \pm 0.004 \\
{[0.595,0.607]}\end{array}$ & $\begin{array}{l}0.615 \pm 0.024 \\
{[0.581,0.664]}\end{array}$ & $\begin{array}{l}0.607 \pm 0.010 \\
{[0.596,0.626]}\end{array}$ & $\begin{array}{l}0.688 \pm 0.024 \\
{[0.665,0.744]}\end{array}$ \\
\hline ML/CS & $\begin{array}{l}1.229 \pm 0.006 \\
{[1.218,1.238]}\end{array}$ & $\begin{array}{l}1.132 \pm 0.029 \\
{[1.100,1.198]}\end{array}$ & $\begin{array}{c}1.281 \pm 0.014 \\
{[1.250,1.319]}\end{array}$ & $\begin{array}{l}1.206 \pm 0.022 \\
{[1.178,1.248]}\end{array}$ \\
\hline PEW/CS & $\begin{array}{l}0.268 \pm 0.007 \\
{[0.254,0.278]}\end{array}$ & $\begin{array}{l}0.324 \pm 0.017 \\
{[0.297,0.346]}\end{array}$ & $\begin{array}{l}0.269 \pm 0.012 \\
{[0.254,0.290]}\end{array}$ & $\begin{array}{l}0.328 \pm 0.013 \\
{[0.299,0.344]}\end{array}$ \\
\hline PPW/CS & $\begin{array}{l}0.458 \pm 0.004 \\
{[0.451,0.463]}\end{array}$ & $\begin{array}{l}0.515 \pm 0.020 \\
{[0.475,0.543]}\end{array}$ & $\begin{array}{l}0.480 \pm 0.007 \\
{[0.468,0.486]}\end{array}$ & $\begin{array}{l}0.522 \pm 0.019 \\
{[0.484,0.550]}\end{array}$ \\
\hline MDL/CS & $\begin{array}{l}0.524 \pm 0.002 \\
{[0.519,0.527]}\end{array}$ & $\begin{array}{l}0.478 \pm 0.005 \\
{[0.470,0.488]}\end{array}$ & $\begin{array}{l}0.531 \pm 0.005 \\
{[0.522,0.538]}\end{array}$ & $\begin{array}{l}0.460 \pm 0.006 \\
{[0.448,0.470]}\end{array}$ \\
\hline MDL & $\begin{array}{c}228 \pm 5 \\
{[219,236]}\end{array}$ & $\begin{array}{c}194 \pm 7 \\
{[182,204]}\end{array}$ & $\begin{array}{c}287 \pm 17 \\
{[259,311]}\end{array}$ & $\begin{array}{c}253 \pm 4 \\
{[247,259]}\end{array}$ \\
\hline $\begin{array}{l}\text { Pearson's } \\
\text { correlation } \\
\text { (MDL-CS) }\end{array}$ & $0.994^{* *}$ & $0.959^{* *}$ & 0.990 ** & 0.525 \\
\hline
\end{tabular}

** 0.01 significance level.

is the smallest in C. obscurior, C. wroughtonii (Fig. 3E), C. minutior, C. tjibodana (Fig. 3A, B), C. kagutsuchi, C. venustula, $C$. batesii and $C$. elegans. Mandibles widen distally, with basal margin roughly parallel with the anterior clypeal margin in full-face view, ventral margin bent ventrad (Fig. 3A, D). Canthellus distinct, almost reaching basal margin, trulleum open, cordiform in dorsolateral view, in dorsal view laterodistally distinctly bordered by basal margin, which is produced as a sharp blade widen- ing towards basal tooth (Fig. 3E). Canthellus, trulleum and mandalus of similar shape in the species examined. Mesal face distally with short longitudinal ridge running towards apical or preapical tooth (Fig. 3G). Another ridge runs beneath teeth along the masticatory margin, ranging from base of preapical tooth to apical margin of basal tooth, (Fig 3F, G). Long setae line this ridge from below (Fig. 3F) and very short setae are present along the distal part of the basal margin. Mesal face distal to canthellus otherwise smooth (no microstructure or setae present).

\section{Male mandibles \\ Cardiocondyla clade A, ergatoid males}

Mandibles without distinct basal and masticatory margins, toothless, generally long and narrow. Mandibles tapering distally (in contrast to worker mandible), curved inwards. Mesal face bulging longitudinally and setose.

\section{C. wheeleri group: C. nigrocerea (Fig. 4)}

Dorsal margin proximally bent ventrally with a distinct obtuse angle (arrow in Fig. 4A-D), mandibles in full-face view oriented anteroventrally. Mandibles narrow, tapering distally to the obtuse angle towards apex. Trulleum distinctly bordered laterodistally by dorsal margin, trulleum not fully visible in lateral view (Fig. 4D). Mesal face with several setae, and with a longitudinal bulge directed towards the apex (Fig. 4E).

\section{C. "argentea" group: C. "argyrotricha" (Fig. 5) (preliminary} names for taxa currently being described by B. Seifert)

Dorsal margin in the distal half and ventral margin bent ventrally (mandible in full-face view directed ventrally) (Fig. 5A, B). Mandibles narrow, tapering in the distal half towards the apex. Dorsal margin proximally roundish instead of forming a distinct ridge, thus canthellus and trulleum fully visible in lateral view; trulleum in dorsal view not

Table 4. Measurement data of workers and males of species of the Cardiocondyla shuckardi, C. batesii and C. elegans species-groups; arithmetic mean \pm standard deviation [range]. CL - cephalic length; CW - cephalic width; ML - mesosoma length; MW - mesosoma width; PEW - width of petiole; PPW - width of postpetiole.

\begin{tabular}{|c|c|c|c|c|c|}
\hline Species & C. venustula & C. venustula & C. batesii & C. batesii & C. elegans \\
\hline Caste & $\begin{array}{l}\text { worker } \\
{[N=10]}\end{array}$ & $\begin{array}{l}\text { ergatoid male } \\
\qquad[N=11]\end{array}$ & $\begin{array}{l}\text { worker } \\
{[\mathrm{N}=7]}\end{array}$ & $\begin{array}{l}\text { ergatoid male } \\
\qquad[N=6]\end{array}$ & $\begin{array}{c}\text { ergatoid male } \\
{[\mathrm{N}=12]}\end{array}$ \\
\hline CS & $\begin{array}{c}560 \pm 14 \\
{[545,582]}\end{array}$ & $\begin{array}{c}641 \pm 26 \\
{[598,671]}\end{array}$ & $\begin{array}{c}505 \pm 4 \\
{[496,509]}\end{array}$ & $\begin{array}{c}512 \pm 19 \\
{[495,545]}\end{array}$ & $\begin{array}{c}548 \pm 18 \\
{[521,577]}\end{array}$ \\
\hline CL/CW & $\begin{array}{l}1.221 \pm 0.015 \\
{[1.194,1.239]}\end{array}$ & $\begin{array}{l}1.197 \pm 0.025 \\
{[1.155,1.249]}\end{array}$ & $\begin{array}{l}1.170 \pm 0.008 \\
{[1.154,1.181]}\end{array}$ & $\begin{array}{l}1.068 \pm 0.028 \\
{[1.034,1.120]}\end{array}$ & $\begin{array}{l}1.031 \pm 0.031 \\
{[0.986,1.103]}\end{array}$ \\
\hline MW/CS & $\begin{array}{l}0.581 \pm 0.008 \\
{[0.570,0.597]}\end{array}$ & $\begin{array}{l}0.742 \pm 0.030 \\
{[0.695,0.785]}\end{array}$ & $\begin{array}{l}0.565 \pm 0.007 \\
{[0.553,0.575]}\end{array}$ & $\begin{array}{l}0.696 \pm 0.023 \\
{[0.665,0.726]}\end{array}$ & $\begin{array}{l}0.670 \pm 0.016 \\
{[0.642,0.694]}\end{array}$ \\
\hline ML/CS & $\begin{array}{c}1.256 \pm 0.021 \\
{[1.220,1.284]}\end{array}$ & $\begin{array}{l}1.273 \pm 0.068 \\
{[1.180,1.380]}\end{array}$ & $\begin{array}{c}1.212 \pm 0.011 \\
{[1.198,1.229]}\end{array}$ & $\begin{array}{c}1.233 \pm 0.024 \\
{[1.202,1.267]}\end{array}$ & $\begin{array}{l}1.188 \pm 0.021 \\
{[1.160,1.232]}\end{array}$ \\
\hline PEW/CS & $\begin{array}{l}0.298 \pm 0.005 \\
{[0.291,0.305]}\end{array}$ & n.a. & $\begin{array}{l}0.282 \pm 0.006 \\
{[0.273,0.292]}\end{array}$ & $\begin{array}{l}0.454 \pm 0.020 \\
{[0.435,0.489]}\end{array}$ & $\begin{array}{l}0.478 \pm 0.016 \\
{[0.448,0.507]}\end{array}$ \\
\hline $\mathrm{PPW} / \mathrm{CS}$ & $\begin{array}{l}0.424 \pm 0.008 \\
{[0.412,0.440]}\end{array}$ & n.a. & $\begin{array}{l}0.519 \pm 0.008 \\
{[0.511,0.531]}\end{array}$ & $\begin{array}{l}0.633 \pm 0.017 \\
{[0.607,0.649]}\end{array}$ & $\begin{array}{l}0.703 \pm 0.023 \\
{[0.670,0.735]}\end{array}$ \\
\hline MDL/CS & $\begin{array}{l}0.534 \pm 0.007 \\
{[0.524,0.545]}\end{array}$ & $\begin{array}{l}0.512 \pm 0.014 \\
{[0.492,0.538]}\end{array}$ & $\begin{array}{l}0.534 \pm 0.003 \\
{[0.530,0.537]}\end{array}$ & $\begin{array}{l}0.487 \pm 0.005 \\
{[0.479,0.494]}\end{array}$ & $\begin{array}{l}0.486 \pm 0.007 \\
{[0.472,0.497]}\end{array}$ \\
\hline MDL & $\begin{array}{c}299 \pm 9 \\
{[290,316]}\end{array}$ & $\begin{array}{c}328 \pm 19 \\
{[296,347]}\end{array}$ & $\begin{array}{c}270 \pm 3 \\
{[264,272]}\end{array}$ & $\begin{array}{c}249 \pm 10 \\
{[237,265]}\end{array}$ & $\begin{array}{c}266 \pm 7 \\
{[255,279]}\end{array}$ \\
\hline $\begin{array}{l}\text { Pearson's correlation } \\
\text { (MDL-CS) }\end{array}$ & $0.901^{* *}$ & $0.892^{\star *}$ & $0.890^{* *}$ & $0.962^{* *}$ & $0.890^{* *}$ \\
\hline
\end{tabular}

** 0.01 significance level; n.a. - morphometric data not available. 

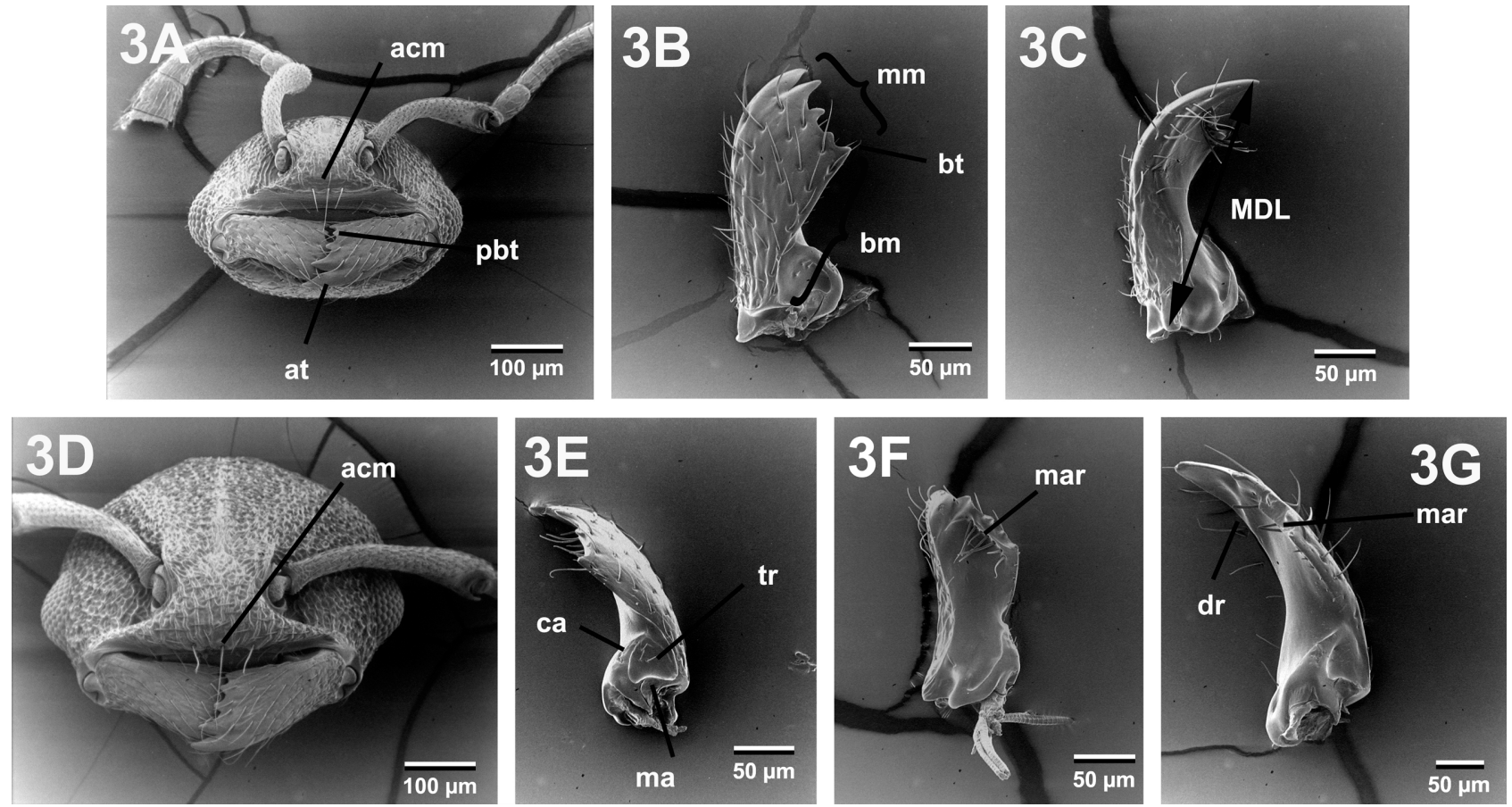

Fig. 3. Worker mandibles. A - C. tjibodana, frontal view of head; B - C. tjibodana, dorso-lateral view of left mandible; C - C. tjibodana, ventral view of right mandible (MDL - mandible length); D - C. "argyrotricha", frontal view of head; $\mathrm{E}-\mathrm{C}$. wroughtonii, dorsal view of right mandible; F - C. wroughtonii, mesal view of right mandible; $G-C$. elegans, dorsomesal view of right mandible. acm - anterior clypeal margin, at - apical tooth, ba - basal angle, bm - basal margin, ca - canthellus, dr - distal ridge, ma - mandalus, mm - masticatory margin, mar - masticatory ridge, $\operatorname{tr}$ - trulleum, pbt - prebasal tooth. In 1A\& D top-bottom corresponds to dorsal-ventral, in 1B, C, E \& F top-bottom corresponds to distal-proximal.
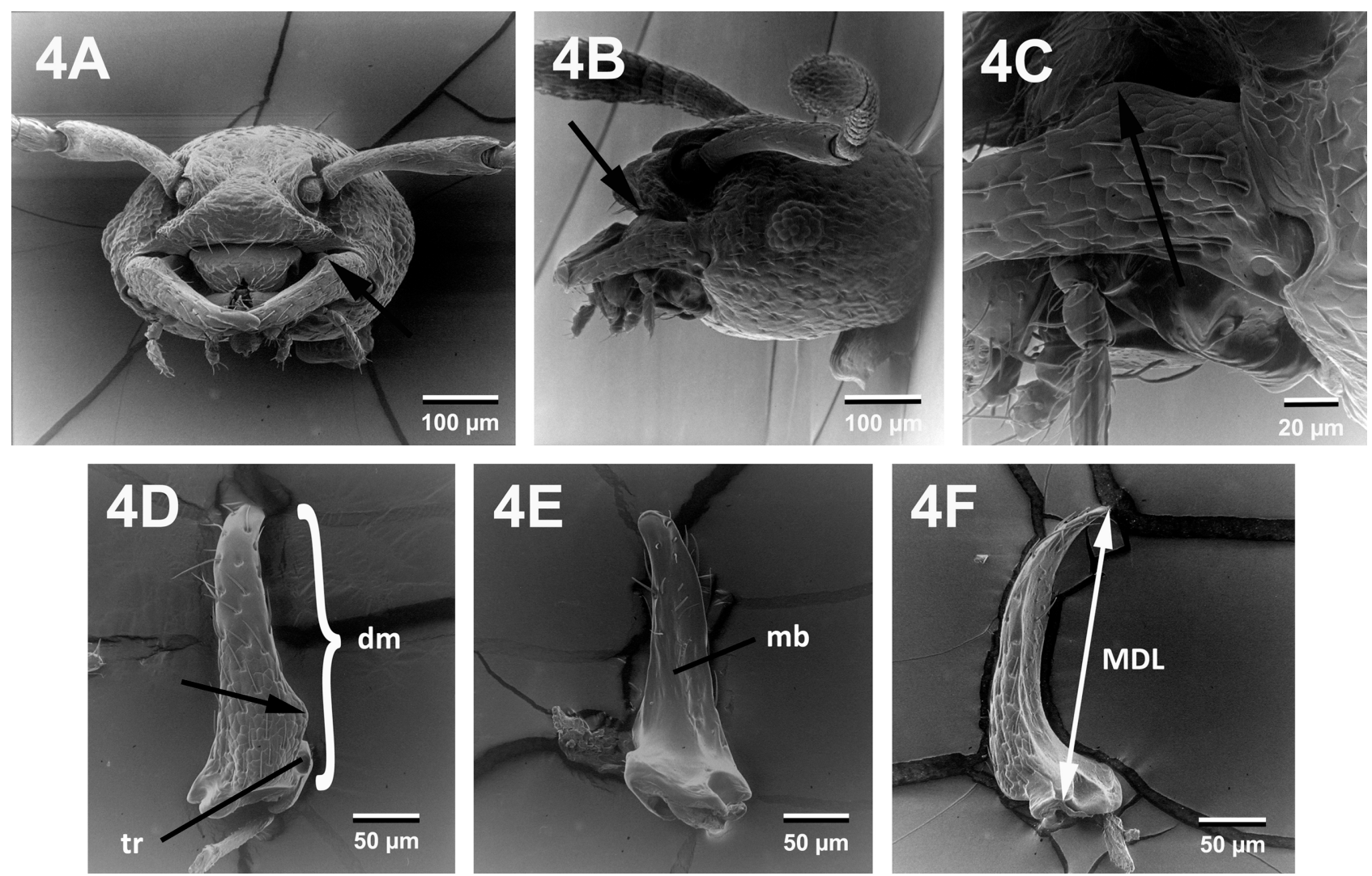

Fig. 4. C. nigrocerea, ergatoid male. Sickle-shaped mandible. A - frontal view of head; B - lateral view of head; C - detail of left mandible in lateral view; D - lateral view of left mandible; $\mathrm{E}$ - dorsomesal view of right mandible; $\mathrm{F}$ - ventral view of right mandible (MDL - mandible length). Arrows indicate angle on dorsal margin. ca - canthellus, dm - dorsal margin, mb - mesal bulge, $\mathrm{tr}$ - trulleum. 

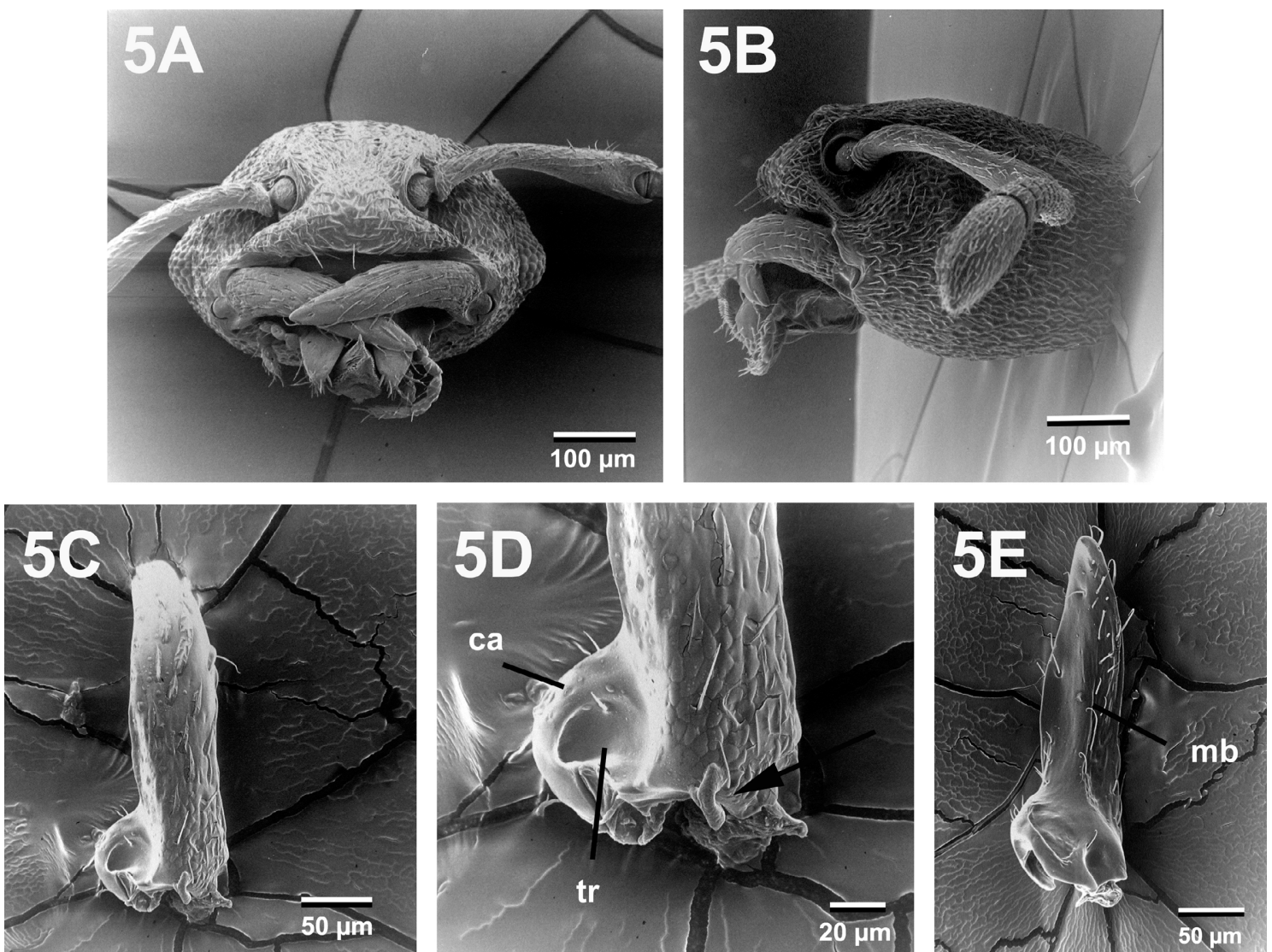

Fig. 5. C. "argyrotricha", ergatoid male. Sickle-shaped mandible. A - frontal view of head; B - lateral view of head; C - dorsomesal view of left mandible; D - lateral view of right mandible; E - detail of right mandible in lateral view showing peculiar structure (arrow); F - mesal view of left mandible. Arrows indicate angle on dorsal margin. ca - canthellus, mb - mesal bulge, $\mathrm{tr}$ - trulleum.
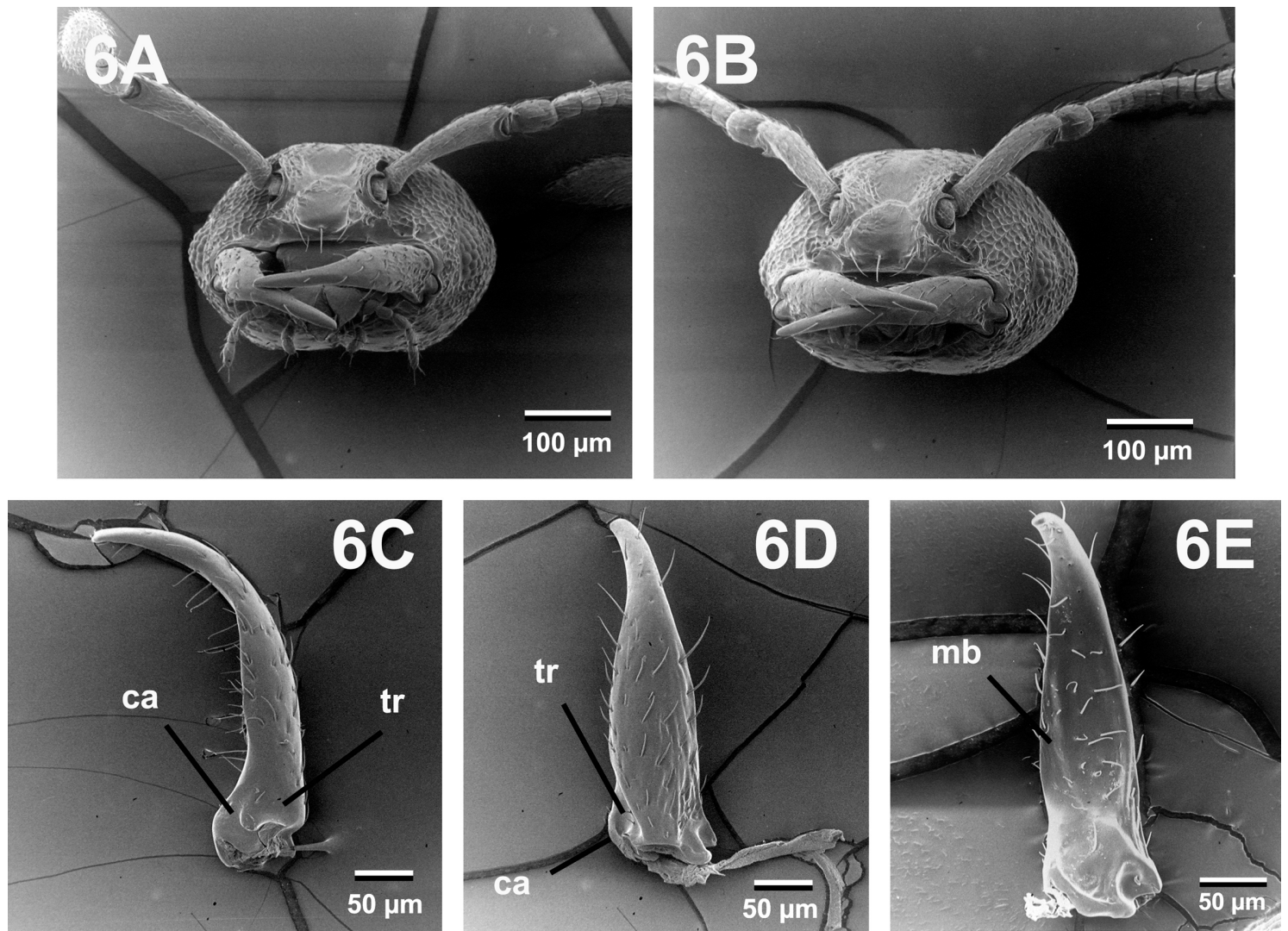

Fig. 6. Ergatoid males of the C. wroughtonii species-group. Sickle-shaped mandible. A - C. wroughtonii, frontal view of head; B - C. obscurior, frontal view of head; $\mathrm{C}-\mathrm{C}$. obscurior, dorsal view of right mandible; $\mathrm{D}-\mathrm{C}$. obscurior, lateral view of right mandible; $\mathrm{E}-\mathrm{C}$. obscurior, mesal view of left mandible. ca - canthellus, $\mathrm{mb}$ - mesal bulge, $\mathrm{tr}$ - trulleum. 

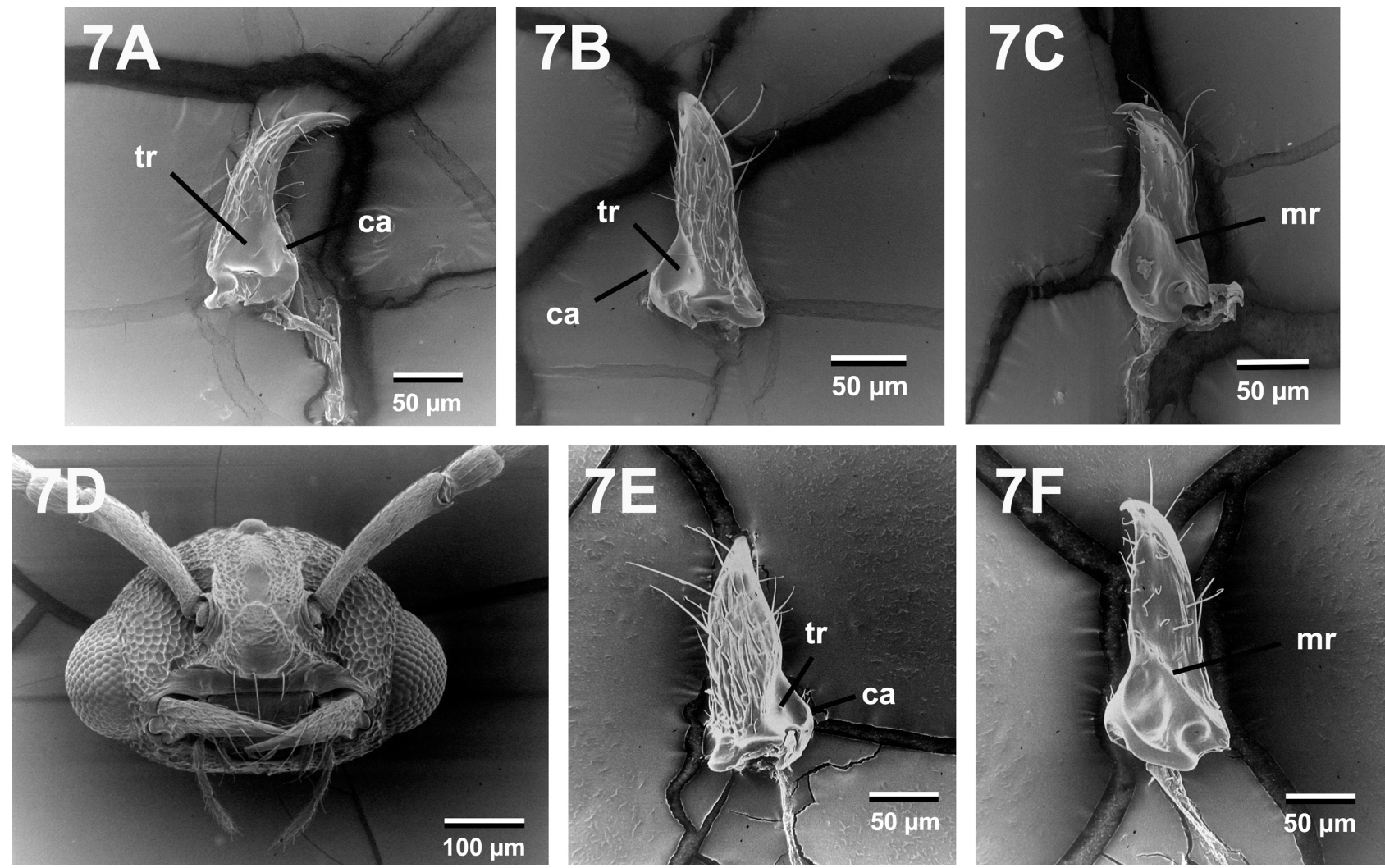

Fig. 7. Winged males of Cardiocondyla clade A. A - C. wroughtonii, dorsal view of left mandible; B - C. wroughtonii, lateral view of right mandible; C - C. wroughtonii, mesal view of left mandible; D - C. obscurior, frontal view of head; E - C. obscurior, lateral view of left mandible; $\mathrm{F}-\mathrm{C}$. obscurior, mesal view of left mandible. ca - canthellus, $\mathrm{mr}-$ mesal ridge, $\mathrm{tr}-\mathrm{trulleum}$.
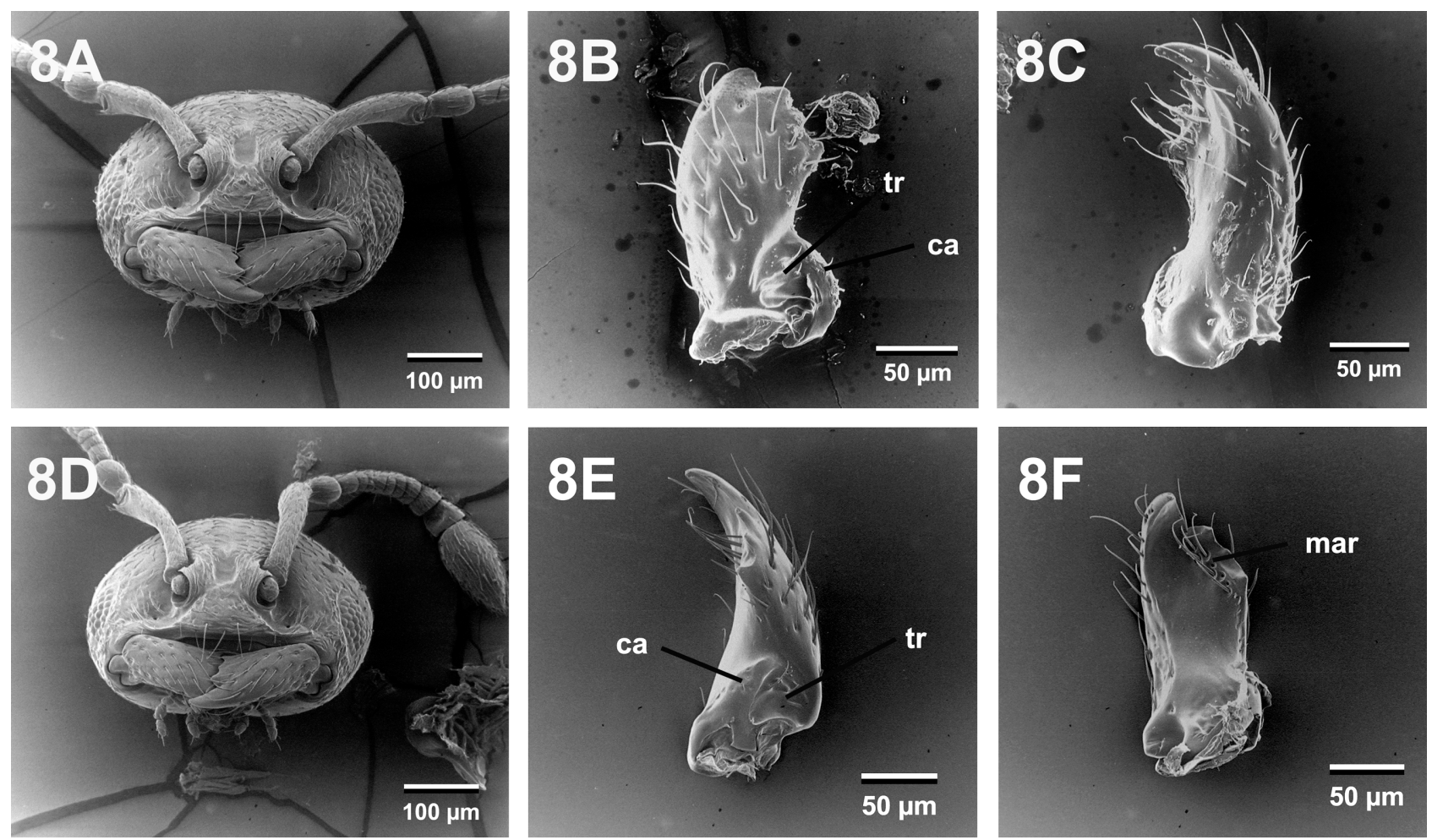

Fig. 8. Ergatoid males of the C. minutior species group. Shear-shaped mandible. A - C. minutior, frontal view of head; B - C. minutior, dorsolateral view of left mandible; C - C. minutior, ventral view of left mandible; D - C. tjibodana, frontal view of head; E - C. tjibodana, dorsal view of right mandible; F - C. tjibodana, mesal view of left mandible. ca - canthellus, mar - masticatory ridge, tr - trulleum. 

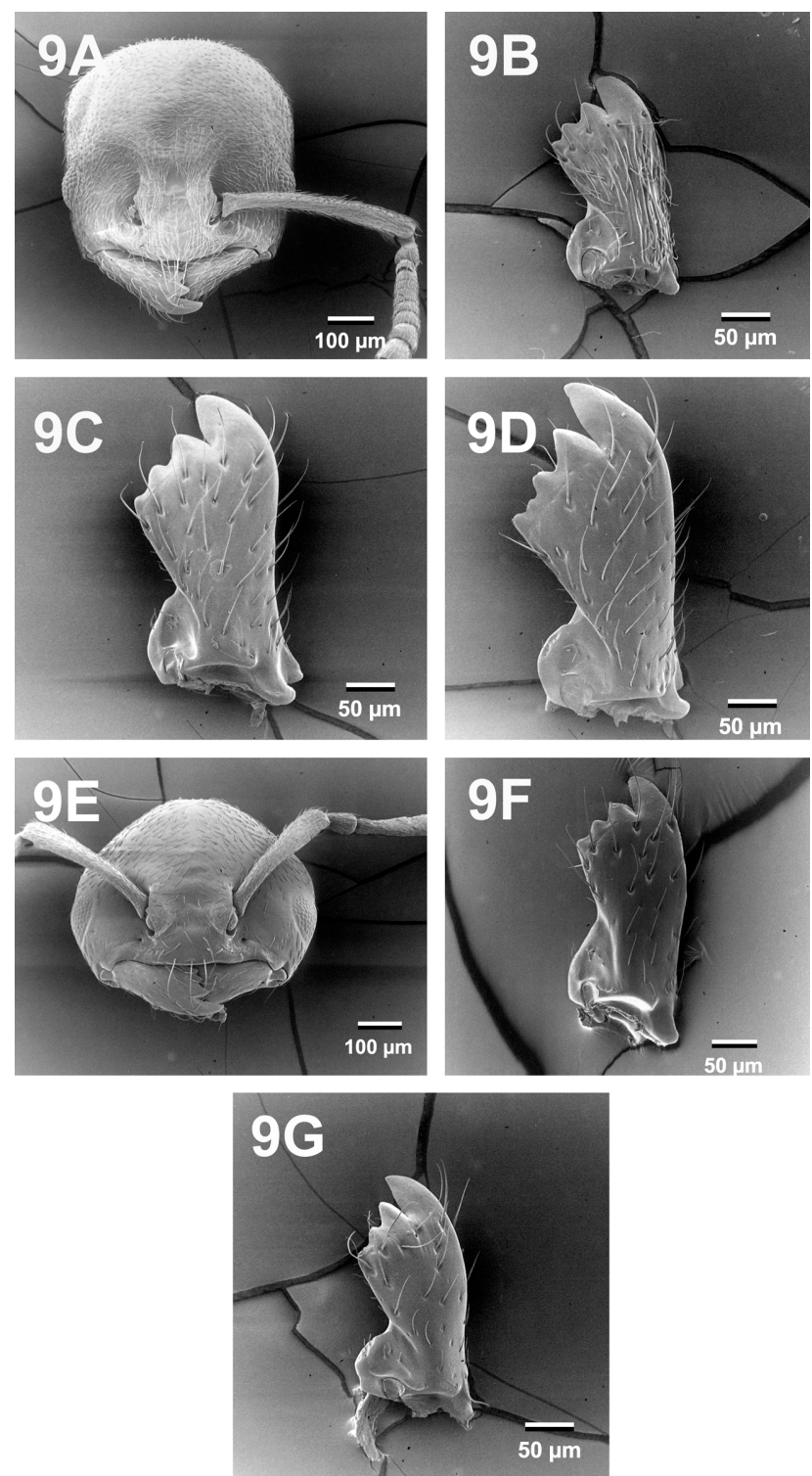

Fig. 9. Ergatoid males of the C. nuda, C. shuckardi, C. batesii, and C. elegans species groups. Shear-shaped mandible. A - C. kagutsuchi, frontal view of head; B - C. kagutsuchi, dorsolateral view of right mandible; $C, D-C$. venustula, dorsolateral view of right mandible; $\mathrm{E}-\mathrm{C}$. elegans, frontal view of head; F - C. elegans, dorsolateral view of right mandible; $G-C$. batesii, dorsolateral view of right mandible.

distinctly bordered laterodistally. Mesal face with a median, longitudinal bulge running towards mandibular apex, dividing the mesal face in two areas; dorsal area smooth, ventral area setose (Fig. 5F). In lateral view, a peculiar sclerotized structure is seen proximally (circle in Fig. 5E).

\section{C. wroughtonii group: C. wroughtonii (Fig. 6A), C. obscurior} (Fig. 6B-E)

Dorsal margin slightly bent ventrad, mandibles in fullface view oriented anteriorly instead of anteroventrally (Fig. 6A, B), narrow, tapering towards the apex, pointed. Distal third notably narrow. Mandibles of $C$. wroughtonii more tapered, as ventral margin is slightly bent dorsally in full-face view (Fig. 6A). Mandibles longer than in the other species studied in clade A. In dorsal view, trulleum not distinctly bordered distolaterally by dorsal margin, which is round, not ridged. Proximal half of mandible with mesal face bulging along dorsal margin (Fig. 6E). Part of mesal face ventral to the bulge is concave and setose (Fig. 6E).

\section{Cardiocondyla clade A, winged males}

C. wroughtonii group: C. wroughtonii (Fig. 7A-C), C. obscurior (Fig. 7E-F)

Mandibles without distinct basal and masticatory margin, toothless, narrowing towards the apex, pointed (Fig. 7A-F). Shorter than those of ergatoid males, yet opposable, curved inwards, in full-face view oriented anteriorly. Trulleum in dorsal view not distinctly bordered distolaterally by dorsal margin (Fig. 7A). Mesal face with a distinct ridge running from proximoventral towards the dorsal margin, reaching it at about half of the mandible length (Fig. 7C, F). Mesal face bearing several setae distal to this ridge (Fig. 7C, F).

\section{Cardiocondyla clade $\mathrm{B}$, ergatoid males}

Mandibles with distinct basal and masticatory margin (Fig. 8A-F, 9A-G); compact in appearance, shorter and distally narrower than the mandibles of workers. Masticatory margin usually with four teeth, some ergatoid males of C. venustula have five teeth (Fig. 9C, D). Apical tooth robust, about twice the size of preapical tooth, teeth decreasing in size from the apex. Trulleum open, in dorsal view distolaterally distinctly bordered by basal margin (Fig. 8E). Presence of ridges on mesal face similar to the mandibles of workers: distally with a short longitudinal ridge extending to the apical or preapical tooth, and another ridge below teeth along the masticatory margin (Fig. $8 \mathrm{C}$ ); otherwise mesal face distal to canthellus flat. Highly ordered arrangement of setae similar to that on the mandibles of workers: long setae arranged along the masticatory margin, short setae along the distal part of the basal margin (Fig. 8F).

Unlike the ergatoid males in clade A, we could not detect any morphological differences between the mandibles of those in clade B.

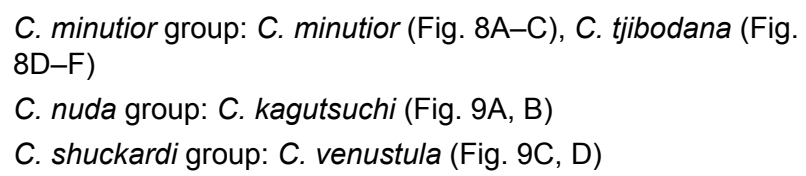

Individuals have mandibles with either 4 or 5 teeth (some individuals with a small, intercalary, prebasal tooth), number of teeth is positively correlated with mandible size (Pearson's correlation: $0.899^{* *}, \mathrm{~N}=11,0.01$ significance level).
C. batesii group: C. batesii (Fig. 9E, F)
C. elegans group: C. elegans (Fig. 9G)

\section{Cardiocondyla clade $\mathrm{B}$, winged males}

C. minutior group: C. minutior (Fig. 10A-C), C. tjibodana (Fig. 10D, E)

Unlike in clade A, where mandibles of winged males are more similar to ergatoid males, those of winged males in clade B resemble the mandibles of workers: distinct basal and masticatory margins, five masticatory teeth with the sub basal one the smallest (as in all workers of the spe- 
Table 5. Summary of qualitative and quantitative morphological data of the mandibles in the examined species.

\begin{tabular}{|c|c|c|c|c|c|}
\hline \multicolumn{3}{|c|}{$\begin{array}{l}\text { Cardiocondyla clade A: } \\
\text { (C. wheeleri, C. "argentea", C. wroughtonii species-groups) }\end{array}$} & \multicolumn{3}{|c|}{$\begin{array}{c}\text { Cardiocondyla clade B: } \\
\text { (C. minutior, C. nuda, C. shuckardi, C. batesii, C. elegans species-groups) }\end{array}$} \\
\hline worker & ergatoid male & winged male & worker & ergatoid male & winged male \\
\hline widening distally & narrowing distally & narrowing distally & widening distally & widening distally & widening distally \\
\hline 5 teeth & toothless & toothless & 5 teeth & $\begin{array}{l}4 \text { teeth ( } 5 \text { in some individuals } \\
\text { of } C . \text { venustula) }\end{array}$ & 5 teeth \\
\hline $\begin{array}{l}\text { mesal face distal to } \\
\text { canthellus flat }\end{array}$ & $\begin{array}{l}\text { mesal face distal to } \\
\text { canthellus bulged }\end{array}$ & $\begin{array}{l}\text { mesal face distal to } \\
\text { canthellus with distinct ridge }\end{array}$ & $\begin{array}{l}\text { mesal face distal } \\
\text { to canthellus flat }\end{array}$ & $\begin{array}{l}\text { mesal face distal } \\
\text { to canthellus flat }\end{array}$ & $\begin{array}{l}\text { mesal face distal } \\
\text { to canthellus flat }\end{array}$ \\
\hline $\begin{array}{l}\text { setae specifically } \\
\text { arranged }\end{array}$ & $\begin{array}{l}\text { setae without specific } \\
\text { distribution }\end{array}$ & $\begin{array}{l}\text { setae without specific } \\
\text { distribution }\end{array}$ & $\begin{array}{l}\text { setae specifically } \\
\text { arranged }\end{array}$ & $\begin{array}{l}\text { setae specifically } \\
\text { arranged }\end{array}$ & $\begin{array}{l}\text { setae specifically } \\
\text { arranged }\end{array}$ \\
\hline \multicolumn{2}{|c|}{ worker MDI < ergatoid male MDI } & n. a. & \multicolumn{2}{|c|}{ worker MDI > ergatoid male MDI } & n. a. \\
\hline MDL/CS $<0.5$ & MDL/CS $>0.5$ & n. a. & MDL/CS > 0.5 & MDL/CS $<0.5$ (except C. venustula) & n. a. \\
\hline \multirow{2}{*}{\multicolumn{2}{|c|}{$\begin{array}{c}\text { worker MDL/CS < ergatoid male MDL/CS } \\
\text { worker } \mathrm{MDL}<\text { ergatoid male MDL }\end{array}$}} & n. a. & \multicolumn{2}{|c|}{ worker MDL/CS > ergatoid male MDL/CS } & n. a. \\
\hline & & n. a. & \multicolumn{2}{|c|}{ worker MDL > ergatoid male MDL (except C. venustula) } & n. a. \\
\hline
\end{tabular}

n. a. - morphometric data not available.

cies in clade B). Trulleum open, in dorsal view distinctly bordered laterodistally by basal margin (Fig. 10B, E). Arrangement of setae and ridges also similar to that on mandibles of workers.

\section{DISCUSSION}

This comparative morphological survey of several species of Cardiocondyla provides morphometric data that reveal how the morphology of ergatoid males differs from that of workers in different species. Seifert (2003) describes several of the differences, namely the increase in the width of the petiolus, postpetiolus and anterior mesosoma. The present study for the first time provides detailed morphometric data confirming the above and additionally reveals that $\mathrm{CL} / \mathrm{CW}$ is generally larger in workers than ergatoid males. This is the case in all the species examined, irrespective of the mating strategy and the clade they are assigned to.

The increase in the width of various body parts is probably correlated with male fighting: ergatoid males use their mandibles to injure or dismember their competitors, with the narrowest body parts being most vulnerable to such attacks. Several species have morphological structures adapted to protect these sensitive regions, such as extensions of the pronotum (Seifert, 2003; Heinze et al., 2010). Interestingly, in the two species examined with non-fighting tolerant ergatoid males (C. batesii, C. elegans), the waist segments (petiolus and postpetiolus) are large com-
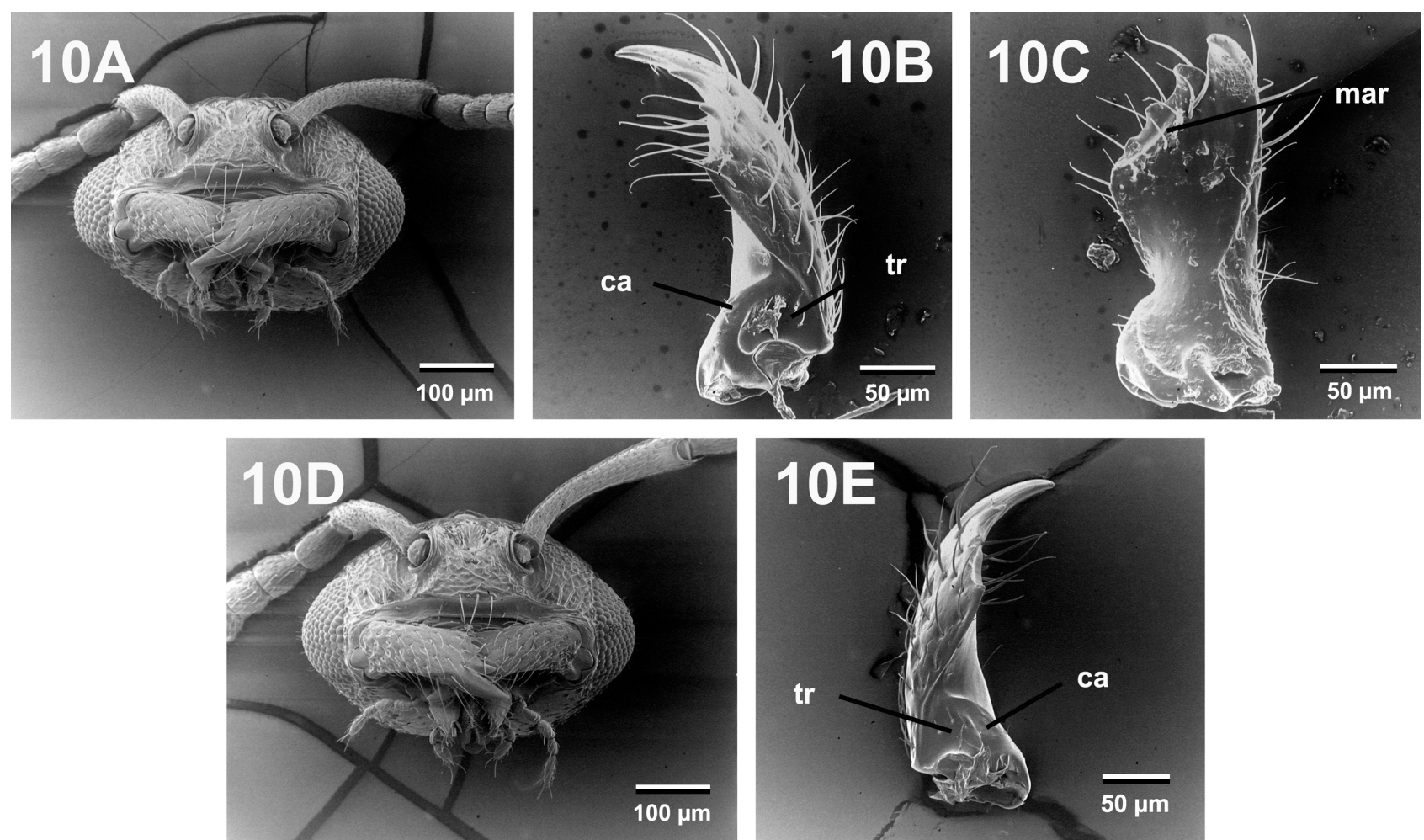

Fig. 10. Winged males of Cardiocondyla clade B. A - C. minutior, frontal view of head; B - C. minutior, dorsal view of right mandible; $C-C$. minutior, mesal view of left mandible; D - C. tjibodana, frontal view of head; E - C. tjibodana, dorsal view of left mandible. ca - canthellus, mar - masticatory ridge, $\mathrm{tr}$ - trulleum. 
pared to those of the workers. This indicates that fighting is an ancestral trait as was previously concluded based on molecular data (Oettler et al., 2010).

Male ant mandibles are typically described as "reduced" and "falcate" (short, incurved, without distinct basal and masticatory margins), "often without function" (Ettershank, 1966; Gotwald, 1969; Boudinot, 2015), although possible adaptations of male mandibles (as of male morphology in general) have only rarely been examined (for exceptions see, e.g., Abell et al., 1999). This is presumably due to the erroneous view that ant males are elusive "sperm delivery vessels" (but see Boomsma et al., 2005; Shik et al., 2012, 2013), only adapted to locate virgin queens and subsequently mate with them. The winged males of $C$. $o b-$ scurior and $C$. wroughtonii have short and curved mandibles, while those of winged males of C. minutior and C. tjibodana are similar to those of workers. In contrast, ergatoid males of Cardiocondyla either have compact four-toothed mandibles with a large apical tooth ("shear-shaped"), or long and narrow ("sickle-shaped") mandibles lacking distinct basal and masticatory margins. The difference in the shape of the mandibles of ergatoid males of species of Cardiocondyla is useful for assigning species to one of the two clades in this genus (Oettler et al., 2010), and mandibular shape (long and narrow vs. four-toothed and worker-like) is clearly correlated with the different ways of killing male competitors in several species in the two clades. Ergatoid males of several species in clade B use their robust mandibles equipped with a large apical tooth to crush the weakly sclerotized cuticle of callow males (Heinze et al., 1993; 1998; Frohschammer \& Heinze, 2009). In contrast, ergatoid males in clade A use their mandibles both to pierce the cuticle of callow males (Kinomura \& Yamauchi, 1987; Stuart et al., 1987) and to grasp a competitor's body and hold it for up to several hours (Kinomura \& Yamauchi, 1987; Yamauchi \& Kawase, 1992; Yamauchi et al., 2006). Mandibles of such ergatoid males are presumably selected to become both longer, to better clasp around wide body parts, and narrower, as this is better for grasping the narrow gap between different body parts, such as between the head and the mesosoma.

Mandible shape and length of ergatoid males are more variable in clade A than in clade B. In clade A, ergatoid males of $C$. obscurior and $C$. wroughtonii are smaller but have both relatively and absolutely longer mandibles than males of C. nigrocerea and C. "argyrotricha". Furthermore, the apices of the mandibles are oriented anteriorly in $C$. obscurior and $C$. wroughtonii but anteroventrally in $C$. nigrocerea and $C$. "argyrotricha". This might reflect different fighting tactics. C. nigrocerea primarily besmears callow males and rarely mature ones (Heinze et al., 2010). Furthermore, casual observations on laboratory colonies indicate that adult ergatoid males of C. "argyrotricha" quickly detect and besmear young rivals (pers. observ.). Freshly eclosed males are less mobile than mature males. They may be easily approached from behind and above and seized by a mature aggressor. In contrast, ergatoid males of $C$. obscurior and C. wroughtonii fight fully sclerotized adult rivals (e.g., Kinomura \& Yamauchi, 1987;
Yamauchi \& Kawase, 1992). Their extremely long, anteriorly oriented mandibles may facilitate seizing and holding mobile mature rivals.

According to Fisher \& Bolton (2016) long and narrow mandibles, without distinct basal and masticatory margins, may have evolved in three ways: (1) "the base of the mandible narrows and the basal angle is obliterated" (basal and masticatory margins forming a single margin), (2) "the masticatory margin becomes elongated and the basal margin contracted", and (3) "the basal margin becomes elongated and the masticatory margin contracted". Ergatoid males of Cardiocondyla clade A have mandibles with few characters and no intermediate conditions, such as mandibles with fewer than five teeth. This makes it difficult to retrace the evolution of this derived type of mandible. Based on the data obtained, we cannot determine if the angle of the dorsal margin corresponds to the basal angle present in worker-like mandibles, or if these structures are different. However, two characteristics appear to be correlated with the formation of narrow mandibles in Cardiocondyla: (1) a bulge or ridge on the mesal surface and (2) unordered distribution of setae (probably derived from the highly ordered setae on triangular mandibles).

Short mandibles are thought to be able to apply greater forces than long mandibles because of the smaller lever arm (Abell et al., 1999). Mandibles of ergatoid males in clade $\mathrm{B}$ are not only relatively shorter than those of males in clade $\mathrm{A}$, but also significantly smaller compared to those of conspecific workers. The mandibles of ergatoid males in clade B retain the overall structure found in workers and therefore are not degenerate as are the short mandibles of many other, short-lived males. In contrast, selection has favoured strong mandibles in those ergatoid males that use them to crush the cuticle of rivals (e.g., Heinze et al., 1993, 1998). Mandibles of the ergatoid males of $C$. minutior and C. kagutsuchi, which exclusively kill young males by crushing their still soft cuticle, have the shortest mandibles relative to head size. We hypothesize that the loss of one masticatory tooth is associated with a reduction in mandible length, as mandibles distally widen to a lesser extent than the mandibles of workers. This assumption is corroborated by the sporadic occurrence of five masticatory teeth on large mandibles of ergatoid males of $C$. venustula. It appears that the prebasal tooth of the worker mandible was reduced during the evolution of the mandibles of ergatoid males, as this tooth is usually the smallest in workers in clade $\mathrm{B}$, and also the smallest on five-toothed mandibles of ergatoid males of $C$. venustula.

Surprisingly our study does not reveal any differences in the shape of mandibles of species in clade B with actively fighting males (C. minutior, C. tjibodana, C. kagutsuchi, $C$. venustula) and those with tolerant, peaceful males $(C$. batesii, $C$. elegans). However, it appears that mandible length of the worker-like mandibles of ergatoid males of species in clade B is correlated with their reproductive strategy. Interestingly, the mandibles of ergatoid males of C. venustula are the longest mandibles (absolute values) in clade B and, in contrast to other species, also absolutely longer than those of conspecific workers. Ergatoid males 
of $C$. venustula kill young rivals by crushing them and also defend small territories by biting, besmearing and holding intruders with their mandibles (Frohschammer \& Heinze, 2009; Jacobs \& Heinze, 2017). As C. venustula is the only species in clade B in which males regularly show this behaviour it cannot be determined whether mandible elongation is similarly associated with besmearing as in clade A.

To conclude, this study adds another piece to the puzzle of the evolution of ant male biology. Detailed observations on the reproductive behaviour of ergatoid males and examination of the size and shape of the mandibles of other species of Cardiocondyla may help to evaluate possible adaptations of the mandibles of ergatoid males and shed light on the evolution of the diverse male traits and reproductive life histories in this fascinating genus of ants.

ACKNOWLEDGEMENTS. We thank S. Buchhauser for developing films, B. Lautenschläger for assisting with microscopy, B. Seifert for the introduction to the measuring technique and $\mathrm{A}$. Bernadou for performing PCA analysis. Two referees and the editor (P. Švácha) made helpful comments on the manuscript. This study was supported by a stipend of Studienstiftung des Deutschen Volkes.

\section{REFERENCES}

Abell A.J., Cole B.J., Reyes R. \& Wiernasz D.C. 1999: Sexual selection on body size and shape in the western harvester ant, Pogonomyrmex occidentalis Cresson. - Evolution 53: 535545.

Boomsma J.J., Baer B. \& Heinze J. 2005: The evolution of male traits in social insects. - Annu. Rev. Entomol. 50: 395-420.

Boudinot B.E. 2015: Contributions to the knowledge of Formicidae (Hymenoptera, Aculeata): a new diagnosis of the family, the first global male-based key to subfamilies, and a treatment of early branching lineages. - Eur. J. Taxon. 120: 1-62.

EtTERSHANK G. 1966: A generic revision of the world Myrmicinae related to Solenopsis and Pheidologeton (Hymenoptera: Formicidae). - Austr. J. Zool. 14: 73-171.

Fisher B.L. \& Bolton B. 2016: Ants of Africa and Madagascar. A Guide to the Genera. University of California Press, Berkeley, 497 pp.

Frohschammer S. \& Heinze J. 2009: Male fighting and "territoriality" within colonies of the ant Cardiocondyla venustula. Naturwissenschaften 96: 159-163.

Gotwald W.H. 1969: Comparative morphological studies of the ants, with particular reference to the mouthparts (Hymenoptera: Formicidae). — Mem. Cornell Univ. Agr. Exp. Sta. 408: $1-150$.

HeInze J. 2017: Life-history evolution in ants: the case of Cardiocondyla. - Proc. R. Soc. (B) 284: 20161406, 8 pp.

Heinze J. \& Hölldobler B. 1993: Fighting for a harem of queens: physiology of reproduction in Cardiocondyla male ants. Proc. Natl. Acad. Sci. USA 90: 8412-8414.

Heinze J., Kühnholz S., Schilder K. \& Hölldobler B. 1993: Behavior of ergatoid males in the ant, Cardiocondyla nuda. - Insectes Soc. 40: 273-282.

Heinze J., Hölldobler B. \& Yamauchi K. 1998: Male competition in Cardiocondyla ants. - Behav. Ecol. Sociobiol. 42: 239-246.

Heinze J., Trindl A., Seifert B. \& Yamauchi K. 2005: Evolution of male morphology in the ant genus Cardiocondyla. - Mol. Phylogenet. Evol. 37: 278-288.
Heinze J., Schmidt C.V., Nugroho H. \& Seifert B. 2010: Wingless fighter males in the Wallacean ant Cardiocondyla nigrocerea (Insecta: Formicidae). — Raffles Bull. Zool. 58: 323-328.

Heinze J., Aumeier V., Bodenstein B., Crewe M. \& Schrempf A. 2013: Wingless and intermorphic males in the ant Cardiocondyla venustula. — Insectes Soc. 60: 43-48.

Heinze J., Seifert B. \& Zieschank V. 2016: Massacre of the innocents in Cardiocondyla thoracica: Manipulation by adult ant males incites workers to kill their immature rivals. - Entomol. Sci. 19: 239-244.

JACoBS S. \& HeInze J. 2017: Between fighting and tolerance: Reproductive biology of wingless males in the ant Cardiocondyla venustula. - Insect Sci. 24: 818-828.

Kinomura K. \& Yamauchi K. 1997: Fighting and mating behaviors of dimorphic males in the ant Cardiocondyla wroughtoni. - J. Ethol. 5: 75-81.

Kugler J. 1983: The males of Cardiocondyla Emery (Hymenoptera: Formicidae) with the description of the winged male of Cardiocondyla wroughtoni (Forel). - Isr. J. Entomol. 17: $1-21$.

Oettler J., Suefuji M. \& Heinze J. 2010: The evolution of alternate reproductive tactics in male Cardiocondyla ants. - Evolution 64: 3310-3317.

SchrempF A. \& HeInZE J. 2007: Back to one: consequences of derived monogyny in an ant with polygynous ancestors. $-J$. Evol. Biol. 20: 792-799.

Schrempf A., Reber C., Tinaut A. \& Heinze J. 2005: Inbreeding and local mate competition in the ant Cardiocondyla batesii. Behav. Ecol. Sociobiol. 57: 502-510.

SeIfert B. 2003: The ant genus Cardiocondyla (Hymenoptera: Formicidae) - a taxonomic revision of the elegans, bulgarica, batesii, nuda, shuckardi, stambuloffi, wroughtonii, emeryi, and minutior species groups. - Ann. Naturhist. Mus. Wien (B) 104: 203-338.

ShiK J.Z., Flatt D., Kay A.D. \& Kaspari M. 2012: A life history continuum in the males of a Neotropical ant assemblage: refuting the sperm vessel hypothesis. - Naturwissenschaften 99: 191-197.

ShiK J.Z., Donoso D.A. \& Kaspari M. 2013: The life history continuum hypothesis links traits of male ants with life outside the nest. — Entomol. Exp. Appl. 149: 99-109.

Stuart R.J., Francoeur R. \& Loiselle R. 1987: Lethal fighting among dimorphic males of the ant, Cardiocondyla wroughtonii. — Naturwissenschaften 74: 548-549.

Terayama M. 1999: Taxonomic studies of the Japanese Formicidae, Part 6. Genus Cardiocondyla Emery. - Mem. Myrmecol. Soc. Jpn. 1: 99-107.

Ward P.S., Brady S.G., Fisher B.L. \& Schultz T.R. 2014: The evolution of myrmicine ants: phylogeny and biogeography of a hyperdiverse ant clade (Hymenoptera: Formicidae). — Syst. Entomol. 40: 51-81.

YAMAUCHI K. \& KaWASE N. 1992: Pheromonal manipulation of workers by a fighting male to kill his rival males in the ant Cardiocondyla wroughtonii. — Naturwissenschaften 79: 274-276.

Yamauchi K., Asano Y., Lautenschläger B., Trindl A. \& Heinze J. 2005: A new type of male dimorphism with ergatoid and short-winged males in Cardiocondyla cf. kagutsuchi. — Insectes Soc. 52: 274-281.

Yamauchi K., Ishida Y., Hashim R. \& Heinze J. 2006: Queenqueen competition by precocious male production in multiqueen ant colonies. — Curr. Biol. 16: 2424-2427.

Received December 18, 2017; revised and accepted July 27, 2018 Published online August 30, 2018 